\title{
Nobelium non-believers
}

\author{
Alfred Nobel's eponymous element, nobelium, was 'first' discovered either in the 1950s or 1960s, in the \\ USSR, Sweden or the USA. Brett F. Thornton and Shawn C. Burdette delve into the ensuing decades of \\ internecine strife over the discovery of element 102.
}

n 1956, a research team in Moscow led by Georgy Flerov bombarded ${ }^{241} \mathrm{Pu}$ with ${ }^{16} \mathrm{O}$ using recently developed heavy-ion beam techniques. The Moscow team may have produced element 102, and contemplated the name joliotium (Jo) to honour 1935 Chemistry Nobelist Irène Joliot-Curie, who had died earlier that year. As Flerov noted later, the early data was inconclusive, and not widely disseminated. Cold War politics may have made joliotium a controversial name elsewhere because Irène Joliot-Curie and her husband were outspoken supporters of the Soviet Union.

In July 1957, researchers working at the Nobel Institute of Physics, now part of Stockholm University, claimed ${ }^{1}$ to have synthesized either ${ }^{251} 102$ or ${ }^{253} 102$ by fusing ${ }^{244} \mathrm{Cm}$ and ${ }^{13} \mathrm{C}$. The team, including collaborators from the UK and Argonne National Laboratory in the US, proposed the name nobelium (No) to recognize the institute's namesake, Alfred Nobel. The name was immediately popularized.

The nobelium report attracted the attention of the superheavy-element group at Lawrence Berkeley Laboratory. After months of unsuccessful attempts to replicate the Stockholm results, the group led by Glenn Seaborg and Albert Ghiorso wondered privately if 'nobelievium' would be a more apt name. Consequently, they initiated new experiments to produce other isotopes of element 102. In 1958 they reported $^{2}$ producing ${ }^{254} 102$ by fusing ${ }^{244} \mathrm{Cm}$ and ${ }^{12} \mathrm{C}$.

By the early 1960s, the Moscow group had moved to the new Joint Institute for Nuclear Research in Dubna. Results with their new accelerator led to suspicions that the Berkeley team had misidentified the reaction product and therefore had claimed incorrectly to have produced ${ }^{254} 102$ in 1958. If an experiment generated an element, but the isotope and/or half-life assignments were incorrect, was the discovery valid? The Dubna group believed

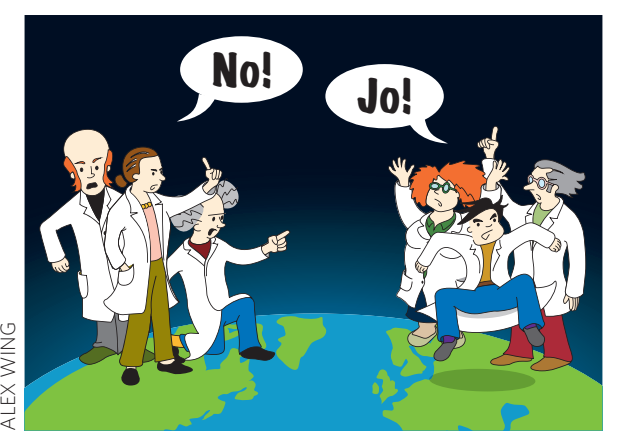

not, declaring IUPAC's acceptance of 'nobelium' hasty, and asserted their discovery of - and preference for - joliotium.

Agitated by the repudiation of their work and competing discovery claim, Ghiorso and colleagues initiated new experiments and re-examined the earlier Berkeley data. They soon realized some of their earlier half-life and isotope identifications had been wrong and the revised analysis of the data aligned more closely with the Dubna findings. In their rebuttal, the Berkeley group emphasized their 'right according to tradition' to name the element, but conceded that they would be content with nobelium ${ }^{3}$. This was ignored in the Soviet Union, where the local discovery of element 102 was lauded officially.

Decades passed without détente. In the early 1990s, spurred by the still unresolved conflict over the names of several superheavy elements, IUPAC re-evaluated the discovery of all transfermium elements. Meanwhile, the Dubna group published ${ }^{4}$ an account of element 102's discovery notably avoiding 'nobelium' and calling the credit given to Berkeley 'groundless' After a long review, IUPAC attributed the definitive discovery of element 102 to two 1966 reports from Dubna ${ }^{5}$. The Berkeley group never relinquished their claims, and accused IUPAC of ex post facto meddling with discovery priority.

Although the Berkeley team contradicted identical reaction and improved methods. Despite the synthesis being the same, the Stockholm group had isolated a product with a different half-life 6 . So, element 102 may have been produced in Stockholm first; but why had they not been able to isolate it convincingly?

Actinide purification protocols that the Stockholm group used to differentiate the common $3+$ actinide ions with cation exchange columns were well established in the 1950s. Unfortunately, $\mathrm{No}^{2+}$ is more thermodynamically stable in aqueous solution ${ }^{7}$; any nobelium produced by the Stockholm group would have eluted at an unexpected, unnoticed time. In hindsight, this provides another example of the predictive power of the periodic table. Analogous to the filled $d$-shell electron configurations of $\mathrm{Cu}^{+}$ and $\mathrm{Ag}^{+}$, promotion of the $6 d^{1}$ electron fills the $5 f$ shell and yields a stable $\mathrm{No}^{2+}$.

No known isotope of element 102 has a half-life greater than the 58 minutes of ${ }^{259} \mathrm{No}$, precluding its inclusion in the famed medals that share the same etymological root. Nevertheless, after all the discord, it's still called nobelium and that is something that will not change over time.

BRETT F. THORNTON is in the Department of Geological Sciences, Stockholm University, 10691 Stockholm, Sweden. SHAWN C. BURDETTE is in the Department of Chemistry and Biochemistry, Worcester Polytechnic Institute, Worcester,

Massachusetts 01609-2280, USA

e-mail: brett.thornton@geo.su.se; scburdette@WPI.EDU

\section{References}

1. Fields, P. R. et al. Phys. Rev. 107, 1460-1462 (1957).

2. Ghiorso, A., Sikkeland, T., Walton, J. R. \& Seaborg, G. T. Phys. Rev. Lett. 1, 18-21 (1958).

3. Ghiorso, A. \& Sikkeland, T. Phys. Today 20, 25-32 (September, 1967).

4. Flerov, G. N. et al. Radiochim. Acta 56, 111-124 (1992).

5. Wilkinson, D. H. et al. Pure Appl. Chem. 65, 1757-1814 (1993).

6. Ghiorso, A., Sikkeland, T. \& Nurmia, M. J. Phys. Rev. Lett. 18, 401-404 (1967).

7. Maly, J., Sikkeland, T., Silva, R. \& Ghiorso, A. Science 160, 1114-1115 (1968) the 1957 Stockholm ${ }^{244} \mathrm{Cm}+{ }^{13} \mathrm{C}$ results in 1958, in 1967 they produced ${ }^{253}$ No using the
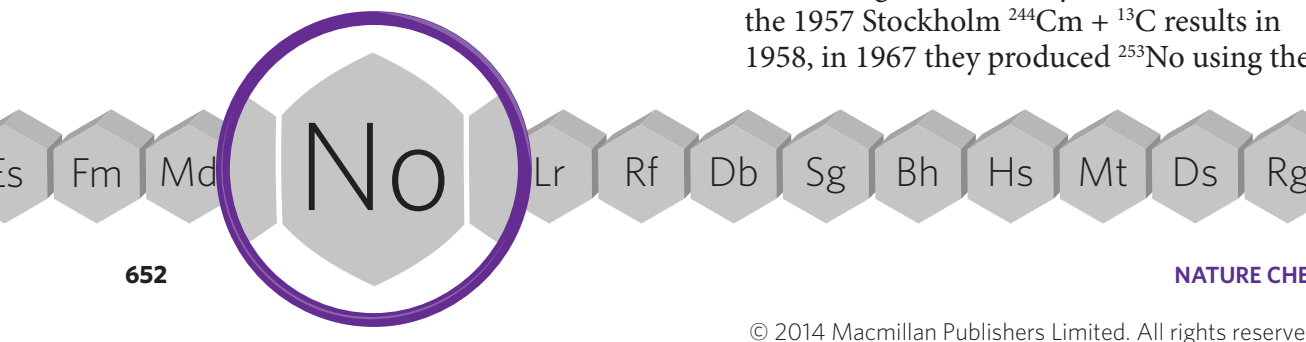